\title{
Stage III Rectal Cancer AJCC v6
}

National Cancer Institute

\section{Source}

National Cancer Institute. Stage III Rectal Cancer A/CC v6. NCI Thesaurus. Code C7792.

Stage III includes: IIIA (T1-T2, N0, M0); IIIB (T3-T4, N1, M0); IIIC (Any T, N2, M0). N1: Metastasis in 1 to 3 regional lymph nodes. N2: Metastasis in 4 or more regional lymph nodes. (AJCC 6th ed.) - 2003 